This leads to a very simple view of malignant tumours and metastasis: malignant cells are cells that can grow in alien environments, and by a process that is not subtle, but largely random accident, they spread, first locally and then eventually through the body to form life-threatening secondary tumours. Metastasis is merely a rare, stochastic event: the chance escape of a cell into the vasculature, its arrival at a suitable site and its growth there.

Much has been made of the need for cells to cross basement membranes, but damage by trauma, inflammation or necrosis can breach basement membranes, and even normal somatic cells can cross vessel walls (S. Koop et al. Proc. Natl Acad. Sci. USA 93, 11080-11084; 1996). Perhaps what is crucial is that when malignant cells are exposed to surrounding connective tissue they simply establish growth in the connective tissue space, instead of helping to repair the tissue organization by closing gaps or going

into apoptosis (programmed cell suicide)

when in the wrong place.

Paul A. W. Edwards

Hutchison/MRC Research Institute,

University of Cambridge, Hills Road,

Cambridge CB2 2XZ, UK

\section{Metastasis: objections to the same-gene model}

\section{Sir - The model of cancer metastasis} suggested by René Bernards and Robert A. Weinberg in their Concepts essay (Nature $\mathbf{4 1 8}, 823 ; 2002$ ) is, in my view, a tautology. The suggestion that the same genes are exclusively responsible both for cancer-cell metastasis and for the emergence and proliferation of cancer cells is tantamount to saying "cancer cells that can proliferate do proliferate". It would be a great loss if this type of idea caused a decline in research to investigate the existence of new genes involved in metastasis, a major factor in cancer mortality.

There is a finite probability that any cancer cell that can proliferate at one body site can also proliferate at other sites, if it can get there and stay there. How these two requirements are met is the real crux of the metastasis question. Bernards and Weinberg wish to dismiss the possibility that specific genetic changes, beyond those that govern proliferation, are required for successful cancer-cell relocation.

However, the argument Bernards and Weinberg used to arrive at this idea is, in my opinion, flawed. They argue that cells that acquire both proliferative and metastatic changes will be rare in primary tumours, thereby making it "difficult to imagine how metastasis can ever proceed". This is a remarkable proposition in the context of a discussion of the initiation of cancer cells, which is itself an extremely rare occurrence. The authors' new concept - that metastasis is not due to a selected cell phenotype - would be better supported by a stochastic mechanism formulation.

Clinically, metastases range from presentation with large primary tumours to presentation without any identifiable primary tumour at all. It may be that the factors responsible for metastasis are cancer-cell proliferation plus myriad other small, unknowable variables that combine to create the conditions for relocation. This is a restatement of the tautology of Bernards' and Weinberg's argument. James L. Sherley

Biological Engineering Division, Massachusetts Institute of Technology, Cambridge, Massachusetts 02139, USA

Bernards and Weinberg reply - We appreciate the interest that our Concepts essay (Nature 418, 823; 2002) has evoked. But Edwards misrepresents our thinking when he writes: "If there is little difference between a primary malignant tumour and its metastases, the crucial issue becomes: what makes a tumour malignant (capable of metastasis)?". In fact, we argued that there are various types of primary tumour, some of which are preordained to become metastatic, others not. Hence, the differences lie between various distinct types of primary tumour. We suppose that in some tumours, the particular combination of alterations/mutations that enables cells to create a robustly growing primary tumour cell population also incidentally empowers them to become metastatic. Also, we do not imply that a single genetic change per tumour is involved in malignancy, as Edwards concludes.

We did not say, as Sherley asserts, that "the same genes are exclusively responsible both for cancer-cell metastasis and for the emergence and proliferation of cancer cells". Instead, we argued that there are multiple alternative genetic pathways that lead to the creation of a primary tumour, each path being defined by the identities of the particular genes that are altered during tumorigenesis. According to our thinking, some combinations of genes that lead to primary tumour formation create growths that are unlikely to metastasize. Other combinations yield tumours that have a high proclivity for metastasizing. In the latter case, the combination of genes that yielded the primary tumour happens to be able to confer invasive/metastatic ability even though these phenotypes were not selected during the clonal expansions that created the primary tumour mass.

We did not say, as Sherley asserts, that cells that acquire both proliferative and metastatic changes will be rare in primary tumours. Instead, we said that certain combinations of genetic alterations that are selected for the proliferative advantage they confer will, incidentally, also confer invasive/metastatic phenotypes. This is in no sense tautological. It is simply the statement of the possibly pleiotropic actions of certain cancer-associated genes. René Bernards, Robert A. Weinberg ${ }^{\star}$ Division of Molecular Carcinogenesis and Center for Biomedical Genetics, The Netherlands Cancer Institute, Plesmanlaan 121, Amsterdam, The Netherlands $\dagger$ Whitehead Institute for Biomedical Research and Department of Biology, Massachusetts Institute of Technology, Cambridge, Massachusetts 02142, USA

\section{Chilean decree will save nights for star-gazers}

Sir - Your editorial "Save starry nights" (Nature 418, 709; 2002) states that Czechoslovakia is the only country with a national policy aimed at limiting light pollution. In fact, Chile, which now contains the largest concentration of optical telescope apparatus in the world, has taken a similar step.

The Association of Universities for Research in Astronomy, the European Southern Observatory and the Carnegie Institution worked for several years with the major Chilean universities and local and national government authorities to implement a strategy for controlling light pollution.

In December 1998, then-President Eduardo Frei signed a decree to establish an environmental norm regulating all outdoor lighting, not only in the areas surrounding the existing observatories, but in all of the II, III, and IV Regions of northern Chile, one-third of the total area of the country. The decree states in part: "The astronomical quality of the skies of the II, III and IV regions of our country constitute a valuable environmental and cultural patrimony recognized internationally as the best existing in the Southern Hemisphere for astronomical observations."

The projected savings in energy costs from replacing polluting lights with wellshielded, energy-efficient ones should more than pay for the initial investment required for the changeover.

Maxine Singer, Augustus Oemler, Mark Phillips

Carnegie Institution of Washington, 1530 P Street NW, Washington, DC 20005, USA 